\title{
Concordancia de la valoración de vía aérea por ultrasonido versus escalas tradicionales y su relación con complicaciones de la intubación orotraqueal
}

\author{
Concordance of ultrasound airway assessment versus traditional scales \\ and its relationship to complications of orotracheal intubation
}

\author{
Jedidiah Cázares Salas, ${ }^{*}$ Carlos Jesús Torres Anaya, ${ }^{\ddagger}$ Marco Antonio García Mora ${ }^{\S}$
}

Citar como: Cázares SJ, Torres ACJ, García MMA. Concordancia de la valoración de vía aérea por ultrasonido versus escalas tradicionales y su relación con complicaciones de la intubación orotraqueal. Acta Med GA. 2021; 19 (4): 501-505. https://dx.doi.org/10.35366/102535

\section{Resumen}

Introducción: La imagen por ultrasonido es una herramienta promisoria para la valoración preanestésica de la vía aérea. Objetivo: Evaluar la concordancia de la valoración de vía aérea por ultrasonido versus escalas tradicionales y su relación con complicaciones de la intubación orotraqueal. Material y métodos: Estudio observacional, transversal, prospectivo realizado en pacientes programados para cirugía bajo anestesia general. En la consulta preanestésica se valoraron con escalas predictoras de intubación difícil (grupo $G[n=16]$ y grupo $T[n=19])$ y en los pacientes del grupo USG se realizaron mediciones ultrasonográficas de intubación difícil. Registrándose durante la intubación la frecuencia de complicaciones. Resultados: La concordancia de hallazgos ultrasonográficos de vía aérea difícil con Mallampati III/ IV y Cormack-Lehane III/IV fue de 43.8-62.5\%. Ocurrieron lesiones en cavidad oral en 0\% del grupo USG y en $15.8 \%$ del grupo $T(p=0.096)$. La incidencia de dolor faríngeo fue $25 \%$ en el grupo USG y $31.6 \%$ en el grupo T ( $p=0.723$ ). Conclusiones: La concordancia de $\mathrm{HH}$ no visible y grosor de la lengua aumentado con escalas tradicionales de vía aérea difícil fue insignificante, pero cuando la vía aérea fue valorada preoperatoriamente con US hubo una tendencia a menor frecuencia de lesiones de la cavidad oral.

Palabras clave: Valoración, vía aérea, ultrasonido, resultados.

\section{Abstract}

Introduction: Ultrasound imaging is a promising tool for preanesthetic assessment of the airway. Objective: To evaluate the concordance of the ultrasound airway assessment versus traditional scales and its relationship with complications during orotracheal intubation. Material y methods: An observational, cross-sectional, prospective study performed in patients scheduled for surgery under general anesthesia. In the preanesthetic consultation, they were assessed with predictive scales of difficult intubation (USG group [ $n=16]$ and T group [n $=19]$ ) and in the patients of the USG group, ultrasonographic measurements of difficult intubation were performed. The frequency of complications was recorded during intubation. Results: The concordance of difficult airway ultrasound findings with Mallampati III/IV and Cormack-Lehane III/IV was $43.8-62.5 \%$. Oral cavity lesions occurred in $0 \%$ of the USG group and in $15.8 \%$ of the T group $(p=0.096)$. The incidence of pharyngeal pain was $25 \%$ in the USG group and $31.6 \%$ in the T group $(p=0.723)$. Conclusions: The concordance of non-visible hyoid bone and increased tongue thickness with traditional difficult airway scales was poor. But when the airway was assessed preoperatively with the US, there was a trend towards a lower frequency of lesions of the oral cavity.

Keywords: Assessment, airway, ultrasound, results.

* Residente tercer año Anestesiología, Nuevo Sanatorio Durango. Facultad Mexicana de Medicina, Universidad La Salle, México.

‡ Anestesiólogo con Alta Especialidad en Anestesia Regional, Nuevo Sanatorio Durango.

$\S$ Jefe de Servicio de Anestesiología, Nuevo Sanatorio Durango.

\section{Correspondencia:}

Dr. Jedidiah Cázares Salas

Correo electrónico: jeczsa@gmail.com

Aceptado: 01-02-2021.

www.medigraphic.com/actamedica 
Abreviaturas:

$\mathrm{HH}=$ Hueso hioides.

SPSS = Paquete estadístico para ciencias sociales (del inglés, Statistical Package for Social Sciences).

ASA = Sociedad Americana de Anestesiólogos (del inglés, American Society of Anesthesiologists).

\section{INTRODUCCIÓN}

La valoración preanestésica es fundamental para identificar a pacientes con mayor probabilidad de vía aérea difícil o identificar condiciones-características de la vía aérea que pudiesen modificar el plan anestésico. ${ }^{1,2}$

Si bien existen métodos y escalas clínicas para identificar a pacientes con vía aérea difícil como la circunferencia de cuello elevada, la clasificación de Mallampati, escala de Patil-Aldreti, la distancia esternomentoniana, la protrusión mandibular y la distancia interincisivos, ${ }^{3,4}$ en los últimos años se ha propuesto el uso del ultrasonido para la valoración de la vía aérea durante la consulta preanestésica, 5,6 en especial porque se han reportado incidencias de 1-5\% de vía aérea difícil no anticipada en pacientes sometidos a intubación orotraqueal. ${ }^{7,8}$

El ultrasonido transcutáneo ofrece la posibilidad de valorar las estructuras de la vía aérea desde la boca hasta el pulmón, identificar patologías y alteraciones anatómicas no diagnósticas, incluso por una valoración clínica minuciosa de vía aérea difícil. ${ }^{9,10}$ Algunos parámetros ultrasonográficos asociados a vía aérea difícil incluyen el grosor del tejido blando de la pared anterior del cuello a nivel del hueso hioides, epiglotis y cuerdas vocales, las distancias mediciones suprahiodeas e infrahiodeas de tejido blando. ${ }^{11,12}$ También se han utilizado mediciones de la lengua como el grosor, el área de la sección transversal, el ancho, el volumen y la relación entre la lengua y la cavidad oral. ${ }^{12}$ Sin embargo, hasta el momento no se han definido parámetros universales de vía aérea obtenidos por ultrasonido que sean predictores de vía aérea difícil. ${ }^{10-12}$ Dos parámetros típicamente utilizados por su fácil obtención son la identificación del hueso hioides $(\mathrm{HH})$ por ultrasonido y un grosor $>3.5 \mathrm{~cm}$ de la lengua. ${ }^{10-12}$ Pero al momento no se conoce su desempeño real para predecir vía aérea difícil ni su concordancia con otros parámetros tradicionales de identificación de vía aérea difícil. ${ }^{13}$

En el presente estudio evaluamos la concordancia de la valoración de vía aérea por ultrasonido con escalas tradicionales y su relación con complicaciones de la intubación orotraqueal.

\section{MATERIAL Y MÉTODOS}

Se presenta un estudio observacional, prospectivo, comparativo en el que se incluyeron pacientes adultos $>18$ años, de ambos sexos, con clasificación ASA I-III que fueron valorados por ultrasonido durante la consulta preanestésica (grupo USG) o que no fueron valorados por ultrasonido durante la consulta preanestésica (grupo T) en el periodo de diciembre de 2017 a febrero de 2018 en el Hospital Sanatorio Durango de la Ciudad de México. Se excluyeron pacientes con tumoraciones de cuello, con antecedente de cirugía de cuello, traqueotomía, o con malformaciones de cabeza y cuello.

De todos los pacientes se registró información demográfica y el resultado de valoración con escalas predictoras de vía aérea difícil incluyendo circunferencia de cuello, la clasificación de Mallampati, escala de Patil-Aldreti, la distancia esternomentoniana, la protrusión mandibular y la distancia interincisivos.

La valoración ultrasonográfica de la vía aérea en el grupo USG se realizó con el paciente en decúbito supino con la cabeza y el cuello en posición neutral utilizando un equipo Mindray M5. Para la identificación del hueso hioides se colocó un transductor lineal de $7.5 \mathrm{MHz}$ y/o un transductor convexo de $3.5 \mathrm{MHz}$ en plano transversal a la línea media para identificar una estructura en forma de $U$ invertida con sombra acústica posterior (hueso hioides). Para la medición del grosor máximo de la lengua se colocó el transductor en el plano coronal justo por detrás del borde posterior del mentón hasta identificar la lengua en su grosor máximo; tras congelar la imagen se midió en cm el grosor de la lengua.

Previo a su realización, el presente estudio fue sometido a aprobación por el Comité de Investigación y Bioética del Hospital Sanatorio Durango. Este estudio se apega a las consideraciones éticas para seres humanos de la Declaración de Helsinki, el Código de Núremberg, el informe de Belmont y a la Ley General de Salud en Materia de Investigación en Salud.

El análisis estadístico se realizó en SPSS v.25 para Mac, en el cual se realizaron pruebas estadísticas descriptivas consistentes en media y desviación estándar para variables cuantitativas así como frecuencias y porcentajes para variables cualitativas. Utilizando tablas de $2 \times 2$, la concordancia se evaluó con la proporción de concordancia $(a+d / n)$ y con el Kappa de Cohen. Como pruebas estadísticas inferenciales se utilizaron t de Student, la $\chi^{2}$ y la exacta de Fisher. Una $\mathrm{p}<0.05$ se considerará significativa.

\section{RESULTADOS}

Se incluyeron 35 pacientes, 16 en el grupo USG y 19 en el grupo T con edad y sexo similar entre grupos. En el grupo USG en $81.3 \%$ de los casos se pudo visualizar la vía aérea y en $87.5 \%$ de los casos el grosor de la lengua fue $>3.5$ cm (Tabla 1). 
Tabla 1: Comparación de características clínicas y ultrasonográficas.

\begin{tabular}{lccc} 
Característica & $\begin{array}{c}\text { Grupo USG } \\
(\mathrm{N}=16)\end{array}$ & $\begin{array}{r}\text { Grupo T } \\
(\mathrm{N}=19)\end{array}$ & $\mathrm{p}^{*}$ \\
\hline Edad (años) & $40.1 \pm 10.2$ & $41.2 \pm 9.8$ & 0.747 \\
$\begin{array}{l}\text { Sexo, } \mathrm{n}(\%) \\
\text { Masculino }\end{array}$ & $13(81.3)$ & $11(57.9)$ & 0.167 \\
$\quad \begin{array}{l}\text { Femenino } \\
\text { Ultrasonografía, } \mathrm{n}(\%) \\
\text { Visualización del }\end{array}$ & $3(18.7)$ & $8(42.1)$ & \\
$\quad$ & $13(81.3)$ & - & - \\
hueso hioides & & & \\
Grosor de lengua $>$ & $14(87.5)$ & - & \\
$3.5 \mathrm{~cm}$ & & &
\end{tabular}

* $\chi^{2}$ o exacta de Fisher cuando $n<5$ por casilla.

USG $=$ ultrasonido, $\mathrm{T}=$ tradicional.

Al evaluar las características de la vía aérea de forma tradicional no se encontraron diferencias significativas entre grupos en la circunferencia de cuello, la clasificación de Mallampati, escala de Patil-Aldreti, la distancia esternomentoniana, la protrusión mandibular ni en la distancia interincisivos, ni en la clasificación Cormack-Lehane durante la laringoscopia (Tabla 2).

En la valoración con escalas tradicionales de evaluación de vía aérea, solamente con Mallampati modificada y Cormack-Lehane se encontraron casos de vía aérea difícil. En el grupo USG 31.3\% tuvieron Mallampati III y IV en comparación con $52.7 \%$ en el grupo $\mathrm{T}(\mathrm{p}=0.306)$, mientras que con Cormack-Lehane $16.8 \%$ en el grupo USG tuvieron vía aérea difícil y $36.9 \%$ en el grupo T ( $p=$ 0.285) (Tabla 2).

La proporción de concordancia de $\mathrm{HH}$ no visible con Mallampati III y IV fue de $50 \%$, y de $\mathrm{HH}$ no visible con Cormack-Lehane III/IV fue de $62.5 \%$. La proporción de concordancia de un grosor de la lengua $>3.5 \mathrm{~cm}$ con Mallampati III/IV y de grosor de la lengua $>3.5 \mathrm{~cm}$, con Cormack-Lehane III/IV fue de $43.8 \%$ en ambos casos (Tabla 3). Todas las laringoscopias fueron directas empleando una hoja Mac 3 o 4. Los dos casos Cormack-IV también se sometieron a laringoscopia directa. En ningún paciente se modificó la técnica de intubación, pero en los casos en los que se anticipaba vía aérea difícil se utilizó almohadilla occipital, guía metálica o una hoja Mac 4.

Al comparar la frecuencia de lesiones de la cavidad oral entre pacientes que tuvieron y no valoración de la vía aérea por ultrasonido durante la consulta preanestésica, se observó una frecuencia de lesiones de la cavidad oral de $15.8 \%$ en el grupo $\mathrm{T}(\mathrm{n}=3)$ y de $0 \%$ en el grupo
USG $(p=0.096)$. En los tres casos en que hubo lesiones de la cavidad oral en el grupo T hubo sangrado (Figura 1). Presentaron dolor faríngeo postoperatorio $25 \%$ de los pacientes del grupo USG y $31.6 \%$ de los pacientes del grupo $\mathrm{T}(\mathrm{p}=0.723)$.

El requerimiento de maniobras adicionales para la intubación también se comparó entre grupos, encontrando que se requirió hiperextensión cervical en $37.5 \%$ del grupo USG y en $57.9 \%$ del grupo T $(p=0.229)$. Se requirió manipulación externa de la laringe en $68.8 \%$ del grupo USG

Tabla 2: Valoración de la vía aérea en la consulta preanestésica y durante la intubación

\begin{tabular}{|c|c|c|c|}
\hline Característica & $\begin{array}{l}\text { Grupo USG } \\
\begin{array}{c}(\mathrm{N}=16) \\
n(\%)\end{array}\end{array}$ & $\begin{array}{c}\text { Grupo T } \\
(\mathrm{N}=19) \\
\mathrm{n}(\%)\end{array}$ & $\mathrm{p}$ \\
\hline $\begin{array}{l}\text { Circunferencia de } \\
\text { cuello }>42 \mathrm{~cm} \\
\text { Mallampati } \\
\text { modificado (clase) }\end{array}$ & $14(87.5)$ & $14(73.7)$ & 0.415 \\
\hline 1 & $5(31.3)$ & $2(10.5)$ & 0.419 \\
\hline II & $6(37.5)$ & $7(36.8)$ & \\
\hline III & $2(12.5)$ & $4(21.1)$ & \\
\hline IV & $3(18.8)$ & $6(31.6)$ & \\
\hline \multicolumn{4}{|l|}{ Patil-Aldreti (grado) } \\
\hline 1 & $6(37.5)$ & $9(47.4)$ & 0.734 \\
\hline II & $10(62.5)$ & $10(52.6)$ & \\
\hline \multicolumn{4}{|l|}{$\begin{array}{l}\text { Distancia } \\
\text { esternomentoniana } \\
\text { (clase) }\end{array}$} \\
\hline I & $12(75.0)$ & $16(84.2)$ & 0.677 \\
\hline ॥ & $4(25.0)$ & $3(15.8)$ & \\
\hline \multicolumn{4}{|l|}{ Protrusión mandibular } \\
\hline $\begin{array}{l}\text { Incisivos inferiores } \\
\text { sobrepasan los } \\
\text { superiores }\end{array}$ & $16(100.0)$ & $18(94.7)$ & 1.000 \\
\hline $\begin{array}{l}\text { Incisivos inferiores } \\
\text { no sobrepasan los } \\
\text { superiores }\end{array}$ & $0(0.0)$ & $1(5.3)$ & \\
\hline \multicolumn{4}{|l|}{$\begin{array}{l}\text { Distancia } \\
\text { interincisivos (grado) }\end{array}$} \\
\hline I & $15(93.8)$ & $0(0.0)$ & 0.457 \\
\hline II & $1(6.2)$ & $0(0.0)$ & \\
\hline \multicolumn{4}{|l|}{$\begin{array}{l}\text { Cormack-Lehane } \\
\text { (clase) }\end{array}$} \\
\hline I & $6(37.5)$ & $5(26.5)$ & 0.719 \\
\hline II & $7(43.8)$ & $7(36.8)$ & \\
\hline III & $2(12.5)$ & $6(31.6)$ & \\
\hline IV & $1(6.3)$ & $1(5.3)$ & \\
\hline
\end{tabular}

USG = ultrasonido, $\mathrm{T}=$ tradicional. 
y en $73.7 \%$ del grupo $\mathrm{T}(\mathrm{p}=0.748)$. Se requirió el uso de guía en $12.5 \%$ del grupo USG y en $10.5 \%$ del grupo $\mathrm{T}$ ( $\mathrm{p}=$ 1.000). Se usó almohadilla occipital en $0 \%$ del grupo USG $y$ en $10.5 \%$ del grupo $\mathrm{T}(\mathrm{p}=0.489)$ (Figura 2$)$.

\section{DISCUSIÓN}

Se ha sugerido que el US en la consulta preanestésica podría ser útil para la evaluación de la vía aérea y la detección de hallazgos que pudiesen anticipar una intubación difícil. Sin embargo, la evidencia hasta el momento es limitada, y se desconoce la asociación de hallazgos ultrasonográficos con escalas predictoras de intubación difícil y con complicaciones de la intubación orotraqueal. ${ }^{14}$ Por ello, en el presente estudio evaluamos la concordancia de la valoración de vía aérea por ultrasonido con escalas tradicionales de vía aérea y su relación con complicaciones durante la intubación.

EI US permite la visualización de la vía aérea desde la boca hasta la tráquea, incluyendo boca, lengua, orofaringe, hipofaringe, hueso hioides, epiglotis y laringe. ${ }^{15}$ Dado que la lengua y el hueso hioides son estructuras fácilmente visualizables y que otras estructuras como la epiglotis son difíciles de visualizar en el plano sagital, ${ }^{16}$ en este estudio evaluamos la visualización del $\mathrm{HH}$ y el grosor de la lengua $>3.5 \mathrm{~cm}$ como potenciales indicadores ultrasonográficos de vía aérea difícil. Sin embargo, al evaluar la concordancia con indicadores tradicionales de vía aérea difícil como la clasificación de Mallampati III/IV y Cormack-Lehane III/IV se observó una concordancia insignificante. De hecho, la mejor concordancia se dio entre $\mathrm{HH}$ no visible y CormackLehane III/IV (62.5\%). Si bien el grosor de la lengua se ha

Tabla 3: Concordancia entre la vía aérea difícil estimada por USG y por escalas de vía aérea tradicionales.

\begin{tabular}{|c|c|c|c|}
\hline Pares & $\begin{array}{c}\text { Proporción de } \\
\text { concordancia } \\
(\%)\end{array}$ & Kарра & $p$ \\
\hline $\begin{array}{l}\text { HH no visible-Mallampati } \\
\text { III/IV }\end{array}$ & 50.0 & 0.306 & 0.509 \\
\hline $\begin{array}{l}\text { HH no visible-Cormack- } \\
\text { Lehane III/IV }\end{array}$ & 62.5 & 0.231 & 0.582 \\
\hline $\begin{array}{l}\text { Grosor de lengua > } 3.5 \\
\mathrm{~cm}-\text { Mallampati III/IV }\end{array}$ & 43.8 & 0.122 & 0.542 \\
\hline $\begin{array}{l}\text { Grosor de lengua }>3.5 \\
\mathrm{~cm}-\text { Cormack-Lehane III/IV }\end{array}$ & 43.8 & 0.064 & 1.000 \\
\hline
\end{tabular}

Figura 1: Comparación de la frecuencia de lesiones y complicaciones de la intubación orotraqueal entre grupos.

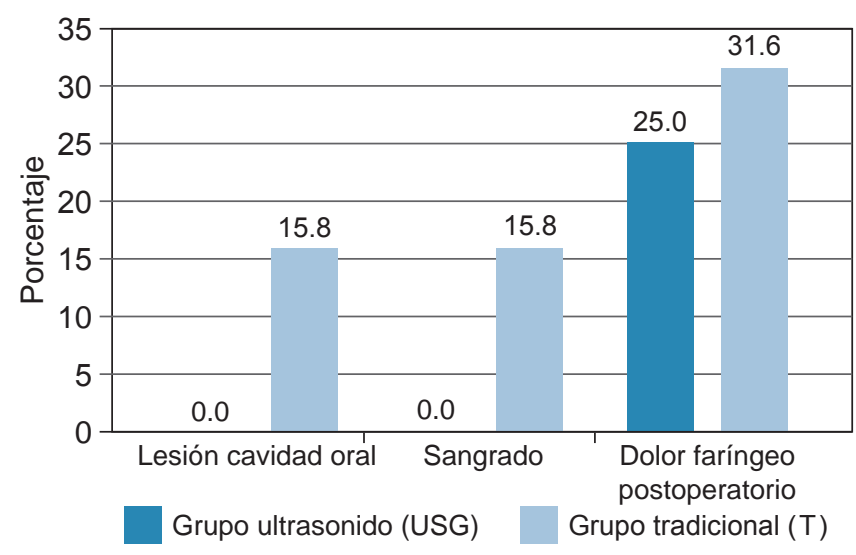

Figura 2: Comparación de requerimiento de maniobras adicionales durante la intubación entre grupos.

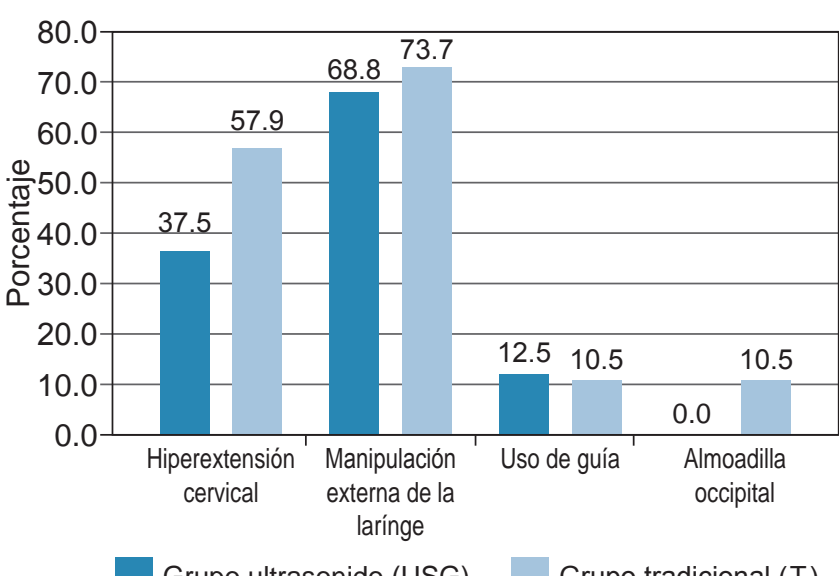

Grupo ultrasonido (USG)

Grupo tradicional $(\mathrm{T})$

propuesto como un predictor independiente de intubación difícil con una sensibilidad y especificidad de 69.7 y $77 \%, 17,18$ en nuestro estudio no encontramos que el grosor de la lengua se hubiese asociado con predictores de intubación difícil, mientras que la no visualización del $\mathrm{HH}$ pudiese ser un mejor indicador de intubación difícil por su mejor concordancia con Cormack-Lehane III/IV. Sin embargo, la concordancia entre éstos sigue siendo insignificante. Un estudio realizado por Bajracharya y colaboradores reveló que la visualización del HH podría ser un interesante indicador de vía aérea difícil, dado que se pudieron ver imágenes claras del $\mathrm{HH}$ en $96.6 \%$ de las intubaciones fáciles, pero no fue posiible visualizar el $\mathrm{HH}$ en $72.7 \%$ de las intubaciones difíciles, es decir, observaron que la sensibilidad y especificidad de la no visualización del HH para vía aérea difícil fue de 73 y 97\%, respectivamente, lo cual no se pudo replicar en nuestro estudio. ${ }^{19} \mathrm{En}$ 
otro estudio en el que se evaluaron mediciones de la vía aérea por US en el preoperatorio y se midieron diversos parámetros incluyendo la visualización del hueso hioides, la distancia de la base de la lengua al hueso hioides, la distancia de la región hiomental, la distancia de la región tirohioidea, la distancia entre la piel y el grosor de la almohadilla grasa al cartílago tiroides, entre muchos más, no se encontró asociada la no visualización del hueso hioides con intubación difícil.

Por lo tanto, es importante en futuros estudios evaluar la utilidad de otros parámetros por US y determinar cuáles parámetros ultrasonográficos son los de mejor desempeño para predecir vía aérea difícil de forma adicional a la detección de anormalidades estructurales o anatómicas de la vía aérea, ya que falta estandarización y recomendaciones basadas en la evidencia para seleccionar los mejores parámetros ultrasonográficos de cabeza y cuello que pronostiquen la vía aérea difícil. ${ }^{20-23}$

\section{CONCLUSIONES}

La concordancia de hallazgos ultrasonográficos de vía aérea difícil (como $\mathrm{HH}$ no visible o grosor de la lengua) con escalas tradicionales de vía aérea difícil (como Mallampati III/IV o Cormack-Lehane III/IV) fue insignificante. Aunque cuando la vía aérea fue valorada preoperatoriamente con US hubo una tendencia a menor frecuencia de lesiones de la cavidad oral. Por lo tanto, es importante identificar hallazgos ultrasonográficos de vía aérea que pronostiquen mejor la vía aérea difícil.

\section{REFERENCIAS}

1. Apfelbaum JL, Connis RT, Nickinovich DG, Pasternak LR, Arens JF, Caplan RA, et al. Practice advisory for preanesthesia evaluation: an updated report by the American Society Of Anesthesiologists task force on preanesthesia evaluation. Anesthesiology. 2012; 116 (3): 522-538.

2. Escobar J. ¿Cuánto podemos predecir la vía aérea difícil?. Rev Chil Anest. 2009; 38: 85-89.

3. Alanís UK, Guerrero MF, Gómez CJR. Relación entre la circunferencia del cuello y vía aérea difícil en pacientes obesos. Anest Mex [Online]. 2017; 29 (2): 18-27.

4. Orozco-Díaz E, Álvarez-Ríos JJ, Arceo-Díaz JL, Ornelas-Aguirre JM. Predicción de intubación difícil mediante escalas de valoración de la vía aérea. Cir Cir. 2010; 78 (5): 393-399.

5. Falcetta S, Cavallo S, Gabbanelli V, Pelaia P, Sorbello M, Zdravkovic I et al. Evaluation of two neck ultrasound measurements as predictors of difficult direct laryngoscopy: A prospective observational study. Eur J Anaesthesiol. 2018; 35 (8): 605-612.

6. Norskov AK, Rosenstock CV, Wetterslev J, Astrup G, Afshari A, Lundstrom LH. Diagnostic accuracy of anaesthesiologists' prediction of difficult airway management in daily clinical practice: a cohort study of 188,064 patients registered in the Danish Anaesthesia Database. Anaesthesia. 2015; 70 (3): 272-281.

7. Norskov AK, Rosenstock CV, Wetterslev J, Lundstrom LH. Incidence of unanticipated difficult airway using an objective airway score versus a standard clinical airway assessment: the DIFFICAIR trial-trial protocol for a cluster randomized clinical trial. Trials. 2013; 14: 347.

8. Heinrich S, Birkholz T, Irouschek A, Ackermann A, Schmidt J. Incidences and predictors of difficult laryngoscopy in adult patients undergoing general anesthesia : a single-center analysis of 102,305 cases. J Anesth. 2013; 27 (6): 815-821.

9. Carrillo-Esper R, Nava-López JA, Romero-Sierra G, Cáñez-Jiménez C. Evaluación ultrasonográfica de la vía aérea superior. Rev Mex Anestesiol. 2014; 37 (2): 123-130.

10. Votruba J, Zemanová P, Lambert L, Vesela MM. The role of airway and endobronchial ultrasound in perioperative medicine. Biomed Res Int. 2015; 2015: 754626.

11. Wojtczak JA. Submandibular sonography: assessment of hyomental distances and ratio, tongue size, and floor of the mouth musculature using portable sonography. J Ultrasound Med. 2012; 31 (4): 523-528.

12. Petrisor C, Dirzu D, Tranca S, Hagau N, Bodolea C. Preoperative difficult airway prediction using suprahyoid and infrahyoid ultrasonography derived measurements in anesthesiology. Med Ultrason. 2019; 21 (1): 83-88.

13. Fulkerson JS, Moore HM, Anderson TS, Lowe RFJ. Ultrasonography in the preoperative difficult airway assessment. J Clin Monit Comput. 2017; 31 (3): 513-530.

14. Kundra P, Mishra SK, Ramesh A. Ultrasound of the airway. Indian J Anaesth. 2011; 55 (5): 456-462.

15. Kristensen MS, Teoh WH, Graumann O, Laursen CB. Ultrasonography for clinical decision-making and intervention in airway management: from the mouth to the lungs and pleurae. Insights Imaging. 2014; 5 (2): 253-279.

16. Singh M, Chin KJ, Chan VW, Wong DT, Prasad GA, Yu E. Use of sonography for airway assessment: an observational study. J Ultrasound Med. 2010; 29 (1): 79-85.

17. Yao W, Wang B. Can tongue thickness measured by ultrasonography predict difficult tracheal intubation? Br J Anaesth. 2017; 118 (4): 601-609.

18. Yadav NK, Rudingwa P, Mishra SK, Pannerselvam S. Ultrasound measurement of anterior neck soft tissue and tongue thickness to predict difficult laryngoscopy - An observational analytical study. Indian J Anaesth. 2019; 63 (8): 629-634.

19. Bajracharya G, Truong A, Truong D, Cata J. Ultrasound-assisted evaluation of the airway in clinical anesthesia practice: past, present and future. Int J Anesth Pain Med. 2015; 1 (2): 1-10.

20. Soltani Mohammadi S, Saliminia A, Nejatifard N, Azma R. Usefulness of ultrasound view of larynx in pre-anesthetic airway assessment: a comparison with Cormack-Lehane classification during direct laryngoscopy. Anesthl Pain Med. 2016; 6 (6): e39566-e39566.

21. Gupta D, Srirajakalidindi A, Ittiara B, Apple L, Toshniwal G, Haber H. Ultrasonographic modification of Cormack Lehane classification for pre-anesthetic airway assessment. Middle East J Anaesthesiol. 2012; 21 (6): 835-842.

22. Jain K, Yadav M, Gupta N, Thulkar S, Bhatnagar S. Ultrasonographic assessment of airway. J Anaesthesiol Clin Pharmacol. 2020; 36 (1): 5-12.

23. Altun D, Kara H, Bozbora E, Ali A, Dinc T, Sonmez S et al. The role of indirect laryngoscopy, clinical and ultrasonographic assessment in prediction of difficult airway. Laryngoscope. 2021; 131 (2): E555-E560. 